\title{
Long-period variable AGB stars in a field towards the galactic bulge *
}

\author{
J.A.D.L. Blommaert ${ }^{1}$, A.G.A. Brown ${ }^{1}$, \\ H.J. Habing ${ }^{1}$, W.E.C.J. van der Veen ${ }^{2}$ and Y.K. $\mathrm{Ng}^{1}$. \\ 1: Sterrewacht Leiden, P.O. Box 9513, 2300 RA Leiden, The Netherlands \\ 2: Columbia University, Dpt. of Astronomy, 538 West $120^{\text {th }}$ St., \\ New York, NY 10027, USA
}

We study two different samples of long-period variable Asymptotic Giant Branch (AGB) stars in a field of low and homogeneous extinction towards the Galactic bulge, the Palomar-Groningen field $\mathrm{Nr}$. 3. The samples were selected to study the evolution of the late phases on the AGB. One sample consists of 486 variables (mostly Miras) optically detected and studied by Plaut (1971) and by Wesselink (1987). The other sample is selected from the IRAS Point Source Catalogue and consists of 239 sources. We made additional infrared measurements between 1.2 and $13 \mu \mathrm{m}$ for a large fraction of both samples. This information was used to identify the IRAS sources and derive the apparent bolometric magnitudes. The samples of Miras and variable IRAS sources have a similar apparent bolometric magnitude distributions, but are displaced by an amount significantly less than expected from the Mira period-luminosity relation (Feast et al. 1989; 0.3 magnitudes as opposed to 0.6 magnitudes). The surface density distribution along the minor axis of the bulge is the same for both samples. We conclude that both samples have evolved from the same parent population and that they represent different evolutionary stages on the AGB. The IRAS sources with the longer periods (on average 450 days (Whitelock et al. 1991) versus on average 250 days for the optical sample) are the further evolved objects. As the IRAS sources have higher mass loss rates we conclude that mass loss increases during the late stages of the evolution. However, we find indications that in some stars the mass loss process has been interrupted for some time; mass loss could be an intermittent process although its overall rate increases in time.

The Miras and the IRAS sources in the bulge have a very similar spatial distribution which also agrees with that found by Blanco (1988) for late type $\mathrm{M}$ giants (Fig. 1). The distribution differs considerably from that of the metal-poor RR Lyrae stars. From model calculations in the literature we estimate that the population of long-period variable AGB stars is more than 10 Gyrs old and originates from stars with a Main Sequence mass of about $1 M_{\odot}$. Unlike Harmon and Gilmore (1988) who estimate the ages

* Based on observations collected at the European Southern Observatory (La Silla, Chile) as part of the ESO Key Programme "Stellar Evolution in the Bulge". 
of the IRAS variables to be less than $5 \mathrm{Gyr}$, we conclude that the ages of the AGB stars are comparable to those of the metal-poor globular cluster population (16 Gyrs).

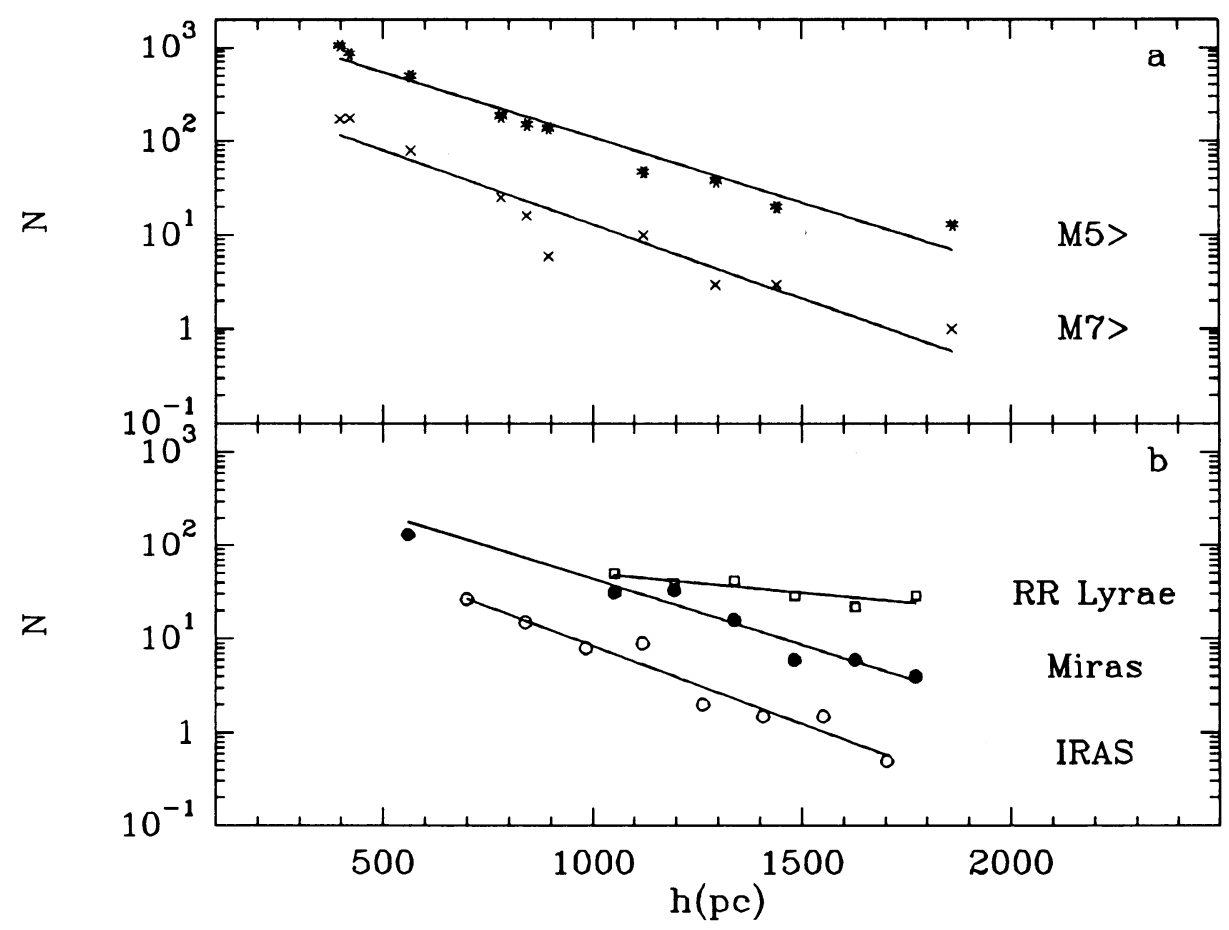

Figure 1. \# a: The projected number density of bulge giants along the $l=0^{\circ}$ axis (Blanco 1988). \# b: The same but now for the RR Lyrae, Miras and IRAS sources (number of sources per 2 square degrees). $R_{o}=8 \mathrm{kpc}$ is assumed.

\section{References}

Blanco, V.M.: 1988, Astron. J. 95, 1400

Harmon, R., Gilmore, G.: 1988, Monthly Notices Roy. Astron. Soc. 235, 1025

Feast, M.W., Glass, I.A., Whitelock, P.A., Catchpole, R.M.: 1989, Monthly Notices Roy. Astron. Soc. 241, 375

Plaut, L.: 1971, Astron. Astrophys. Supplem. Series 4, 75

Whitelock, P.A., Feast, M.W., Catchpole, R.M.: 1991, Monthly Notices Roy. Astron. Soc. 248, 276

Wesselink, T.J.H.: 1987, Ph.D. Thesis, Catholic University of Nijmegen 